\title{
Atrito e Desgaste de Recobrimentos de PET, Politeraftalato de Etileno, Pós-consumo Processados por Aspersão Térmica
}

\author{
Rogério A. X. Nunes, Wagner Sade \\ REDEMAT, CETEC,UFOP-UEMG
}

\author{
José Roberto T. Branco \\ Fundação Centro Tecnológico de Minas Gerais - CETEC, UFOP, REDEMAT
}

Resumo: A aspersão térmica envolve processos de recobrimentos que podem utilizar materiais cerâmicos, poliméricos, metálicos ou misturas destes. O material a ser depositado é fundido total ou parcialmente. As partículas aquecidas são aceleradas e projetadas em direção a uma superfície devidamente preparada, onde se formam camadas com estrutura lamelar. Os efeitos das variáveis do processo de aspersão, pressão dos gases de combustão, taxa de alimentação e gás de arraste no transporte do material, sobre a resistência ao desgaste e atrito de filmes PET foram investigados. Os recobrimentos de PET foram caracterizados através do desgaste micro-abrasivo, utilizando o ensaio de caloteste, e da medida dos coeficientes de atrito, com o ensaio de pino-sobre-disco. Os valores do coeficiente de desgaste abrasivo e do coeficiente de atrito dos recobrimentos são comparados com os valores da garrafa PET utilizada como referência. Os coeficientes de desgaste encontrados foram similares aos valores de garrafas PET da ordem de $10^{-5}$. As análises estatísticas dos resultados deste trabalho indicam que os efeitos das interações das três variáveis, pressão dos gases de combustão, taxa de alimentação e gás de arraste foram significativos para o coeficiente de desgaste e não significativos para o coeficiente de atrito.

Palavras-chave: Aspersão térmica, PET, tribologia.

\section{Friction and Wear of a Thermal Sprayed PET - Poly(Ethylene Teraphthalate) Coating}

\begin{abstract}
The thermal spray technique involves coating processes that can use ceramic, polymers, metallic materials or a blend of these. The material to be deposited is melted totally or partially, where the heated particles are accelerated and projected toward a prepared surface forming layers with a lamellar structure. The effect of the spray process parameters such as, combustion pressure, feed rate and carrier gas, on the wear resistance and friction of PET films was evaluated. The PET coatings were characterized by measuring the wear coefficient through the calowear type testing and the friction coefficients using pin-on-disk testing. The values of the abrasive wear coefficient and the friction coefficient of the coatings were compared to the values of the bottle PET reference sample. The wear coefficient values were similar to the values for the PET bottles, in the range of $10^{-5}$. The statistical analyses of the results indicate that the interaction effects of the three variables, gas pressure, feeding rate and carrier gas were significant for the wear coefficient but not for the friction coefficient.
\end{abstract}

Keywords: Thermal spray, PET, tribology.

\section{Introdução}

A técnica da aspersão térmica, dentro da Engenharia de Superfícies, mantém-se como uma das suas promissoras alternativas para a produção de novos materiais com características para atender as crescentes exigências do mercado em relação às suas propriedades físicas, químicas e tribológicas.

O papel da aspersão térmica no avanço tecnológico tem experimentado reconhecimento crescente. A aspersão térmica é um processo que foi usado inicialmente na manutenção de equipamentos da indústria pesada e aeroespacial. Devido à diversidade de camadas protetoras, muitas empresas têm reconhecido a versatilidade e economia inerentes deste processo, tendo introduzido esta tecnologia no ambiente industrial ${ }^{[1]}$.

Como são usados para proteger superfícies contra umidade, corrosão e produtos químicos agressivos, os recobrimentos poliméricos por aspersão térmica adquiriram um significado importante para muitas indústrias do ramo petroquímico, automotivo e da aviação. Uma das maiores vantagens desse processo comparado a outras técnicas, é que com esta os recobrimentos podem ser aplicados sem restrição quanto às dimensões da superfície a serem recobertas.

A aspersão apresenta grande versatilidade, decorrente da larga faixa de materiais que podem ser depositados, além de diferentes formas e tamanhos de substratos. No atual estado

Autor para correspondência: José Roberto T. Branco, Fundação Centro Tecnológico de Minas Gerais, Setor de Tecnologia Metalúrgica, Laboratório de Engenharia e Modificações de Superfícies, Av. José Candido Silveira 2000, CEP: 31170-000, Belo Horizonte, MG, Brasil. E-mail: jose.branco@cetec.br 
da arte, poucos polímeros têm sido testados como recobrimen$\operatorname{tos}^{[2,3]}$. É recomendável que se desenvolvam, para cada polímero, alguns parâmetros críticos de processos, os quais devem ser selecionados adequadamente a fim de se garantir máxima fusão e mínima degradação das partículas. Esses parâmetros de aspersão deve se adequar a algumas características do pó, principalmente as referentes à composição química, morfologia, fluidez, distribuição de massa molar e ao tamanho de partículas ${ }^{[3,4]}$.

Dentre os parâmetros do processo ou suas variáveis destacam-se: 1) variáveis de alimentação: tipo, tamanho e forma do pó; fluxo e velocidade do gás de arraste; 2) variáveis da tocha: fluxo e composição dos gases; temperatura da tocha; resfriamento; energia térmica; 3) variáveis da aspersão: velocidade e temperatura das partículas; trajetória das partículas até a tocha; energia e ângulo de impacto; estado de solidificação; morfologia; e 4) variáveis do substrato: tipo e temperatura; distância de aspersão $0^{[5]}$.

Zhang ${ }^{[6]}$ descreve que os parâmetros de processo da aspersão têm efeito significativo sobre o aquecimento, fusão e degradação das partículas aspergidas na chama. A aspersão afeta a estrutura molecular das cadeias poliméricas, o que exerce influência sobre as propriedades do recobrimento formado.

Os materiais poliméricos em seu volume ou em filmes são utilizados em aplicações onde ocorre deslizamento e por isso muitos estudos são dedicados a sua resistência ao desgaste ${ }^{[7,8]}$. Comparados com metais e cerâmicas, os polímeros exibem menores coeficientes de atrito, com valores normalmente entre 0,1 e 0,5 , por isso são utilizados em aplicações tribológicas usualmente deslizando contra contracorpos mais duros ${ }^{[9]}$.

Briscoe e Sinha ${ }^{[10]}$ desenvolveram modelos matemáticos envolvendo adesão e abrasão com o objetivo de interpretar a origem do atrito e do desgaste.

O presente trabalho busca contribuir para o desenvolvimento de recobrimentos por aspersão térmica, a partir de PET pós-consumo, poli(tereftalato de etileno) em tubulações de aço carbono onde se requer melhor comportamento tribológico.

Com isto espera-se contribuir para o desenvolvimento de tecnologias que permitam reciclar materiais mais facilmente, protegendo o meio ambiente e também, desenvolver os mercados para os produtos reciclados.

Inicialmente fez-se um estudo da influência dos parâmetros do processo de aspersão térmica e seus efeitos nas propriedades tribológicas dos recobrimentos desse polímero empregando-se a técnica de planejamento de experimentos. Foram encontrados nesses recobrimentos baixos coeficientes de atrito e resistência ao desgaste. $\mathrm{O}$ estudo da resistência ao desgaste e do coeficiente de atrito serão objetos de estudo deste trabalho.

\section{Materiais e Métodos}

\section{Matéria prima}

A matéria prima utilizada neste trabalho tem sua origem nas garrafas de refrigerante (coca-cola) pós-consumo. A Fi-

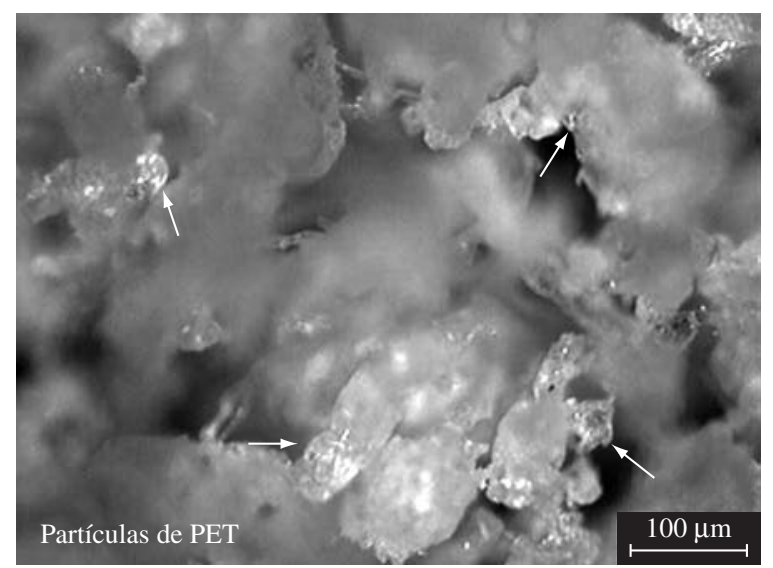

Figura 1. Partículas do pó de PET pós-consumo.

gura 1 apresenta imagem das partículas do pó de PET. O formato irregular é decorrente do processo de cominuição para a produção do pó de PET, técnica desenvolvida pelo CETEC ${ }^{[11]}$. O tamanho das partículas usado situa-se entre 65 a 100 mesh. Lâminas de garrafa de refrigerante PET pós-consumo foram usadas como referência.

\section{Produção de recobrimentos}

O sistema de aspersão térmica à combustão utilizado neste trabalho é constituído de quatro unidades principais: pistola, unidade de alimentação de pó, unidade de controle e medida de fluxo de gases e unidade de controle do fluxo de ar. As pressões dos gases utilizados nesse processo - propano, oxigênio, nitrogênio e ar comprimido - são controladas através de válvulas conectadas diretamente nos cilindros de armazenamento.

Chapas de aço ABNT 1020 foram utilizadas como substrato. A Tabela 1 apresenta alguns dos parâmetros de aspersão. Realizou-se um planejamento fatorial completo, $2^{3}$, com os fatores pressão dos gases de combustão, taxa de alimentação em g/min e tipo de gás de arraste no transporte do material. Os recobrimentos foram realizados em ordem aleatória.

A Tabela 2 mostra os parâmetros de processo utilizados na confecção das amostras, apresentando as medidas da espessura do recobrimento e a temperatura no fim do processo, medida com um pirômetro infravermelho.

Tabela 1. Parâmetros de aspersão.

\begin{tabular}{lc}
\hline \multicolumn{1}{c}{ Parâmetros } & Valores \\
\hline Distância de aspersão $(\mathrm{mm})$ & 30 \\
Pressão dos gases de combustão (psi) & 40 e 50 \\
Medida de razão do fluxo (FMR) & 30 \\
Taxa de alimentação $(\mathrm{g} / \mathrm{min})$ & $2 \mathrm{e} 4$ \\
Temperatura de pré-aquecimento $\left({ }^{\circ} \mathrm{C}\right)$ & 100 \\
Gás de arraste do alimentador de pó & ar comprimido \\
& e nitrogênio \\
Rugosidade superficial $(\mu \mathrm{m})$ & 3,0 \\
\hline
\end{tabular}


Tabela 2. Parâmetros das amostras: a pressão dos gases de combustão (P), a taxa de alimentação em $\mathrm{g} / \mathrm{min}$ e o tipo de gás de arraste do pó (nitrogênio ou ar comprimido).

\begin{tabular}{cccccc}
\hline Amostra & $\begin{array}{c}\mathbf{P} \\
(\mathbf{p s i g})\end{array}$ & $\begin{array}{c}\text { Taxa } \\
(\mathbf{g} / \mathbf{m i n})\end{array}$ & Gás & $\begin{array}{c}\mathbf{T} \\
\left({ }^{\circ} \mathbf{C}\right)\end{array}$ & $\begin{array}{c}\text { Espessura } \\
(\mathbf{m m})\end{array}$ \\
\hline 1 & 40 & 2 & $\mathrm{~N}$ & 237 & 0,20 \\
2 & 40 & 2 & $\mathrm{Ar}$ & 223 & 0,20 \\
3 & 50 & 2 & $\mathrm{Ar}$ & 199 & 0,23 \\
4 & 50 & 2 & $\mathrm{~N}$ & 199 & 0,21 \\
5 & 40 & 4 & $\mathrm{~N}$ & 179 & 0,51 \\
6 & 40 & 4 & $\mathrm{Ar}$ & 195 & 0,45 \\
7 & 50 & 4 & $\mathrm{~N}$ & 200 & 0,40 \\
8 & 50 & 4 & $\mathrm{Ar}$ & 201 & 0,49 \\
\hline
\end{tabular}

\section{Ensaio de desgaste}

O desgaste abrasivo foi determinado no ensaio caloteste que promove a interação de uma esfera de aço em rotação com a superfície da amostra, em contato com um abrasivo, a alumina, que forma uma cratera semi-hemisférica, a partir da qual, através da medida de suas dimensões calcula-se a taxa de desgaste do recobrimento através das Equações 1, 2 e 3.

$$
\begin{aligned}
& S=2 . \pi \cdot R \cdot n \\
& V=\frac{\pi \cdot D^{4}}{64 \cdot R} \\
& Q=\frac{V}{S}
\end{aligned}
$$

sendo $D$ o diâmetro da cratera; $n$ o número de voltas; $R$ o raio da esfera; $V$ o volume desgastado; $S$ a distância percorrida; e $Q$ a taxa de desgaste ${ }^{[9]}$.

O coeficiente de desgaste de um material $(\mathrm{K})$ é calculado usando-se a equação de Archard (Equação 4) sendo, W a carga aplicada e $\mathrm{H}$ a dureza.

$$
Q=\frac{K W}{H}
$$

A distância percorrida pela esfera foi de 100, 200, 300, 500 e 1000 voltas e a carga aplicada de $13,7 \mathrm{~N}$ se manteve constante. Realizaram-se três medidas por amostra e por condição de ensaio.

\section{Medidas de coeficiente de atrito}

Foram realizados ensaios de pino-sobre-disco (PSD), sendo os pinos esferas de aço cromo e os discos de revestimentos poliméricos. Neste trabalho os objetivos do ensaio foram determinar o coeficiente de atrito e identificar as características do desgaste por deslizamento quanto a transferência de massa do filme para a superfície da esfera. Os parâmetros utilizados no ensaio foram de $10 \mathrm{~N}$ de carga, $10 \mathrm{rpm}$ para a rotação da esfera sobre os discos, $14 \mathrm{~mm}$ de diâmetro de trilha com 50 voltas, totalizando uma distância de $2200 \mathrm{~mm}$. A limpeza das amostras e das esferas foi realizada com água e detergente e posteriormente com água destilada e álcool etílico para enxágüe e secagem. As amostras foram pesadas antes e depois dos testes para efeito de perda de massa.

\section{Resultados e Discussão}

A Figura 2 mostra a seção transversal do recobrimento. Esta imagem representa a formação do recobrimento de uma estrutura lamelar na superfície e um coalescimento na interface explicado pela temperatura e transferência de calor filme/substrato.

A Tabela 3 apresenta os resultados do coeficiente de atrito e do coeficiente de desgaste abrasivo das oito amostras recobertas por aspersão térmica de acordo com a matriz de experimentos e o resultado de uma amostra de referência representada por uma lâmina da garrafa de PET pós-consumo.

O gráfico da Figura 3 representa a variação do volume da cratera em função da distância percorrida pela esfera em $\mathrm{mm}$. O coeficiente angular da reta é a taxa de desgaste da amostra 1 ou $\mathrm{Q}=3 \times 10^{-6} \mathrm{~mm}^{3} / \mathrm{mm}$.

Shipway et $a^{[12]}$ encontraram valores do coeficiente de desgaste microabrasivo (K) em PETG em torno de $8,5 \times 10^{-5} \mathrm{~mm}^{3} / \mathrm{N}$.mm. Friedrich et al ${ }^{[13]}$ mostram valores de desgaste em compósitos poliméricos, tendo obtido valores da taxa de desgaste $(\mathrm{Q})$ de alguns compósitos, tais como, teflon

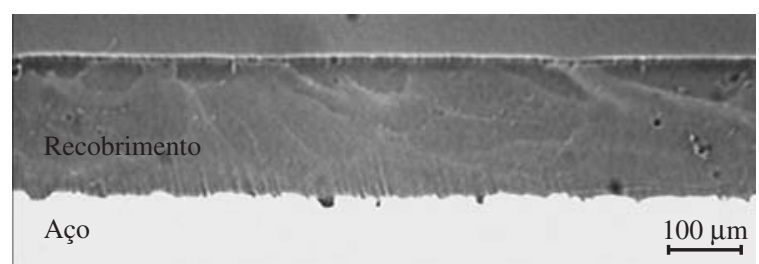

Figura 2. Seção transversal do recobrimento de PET.

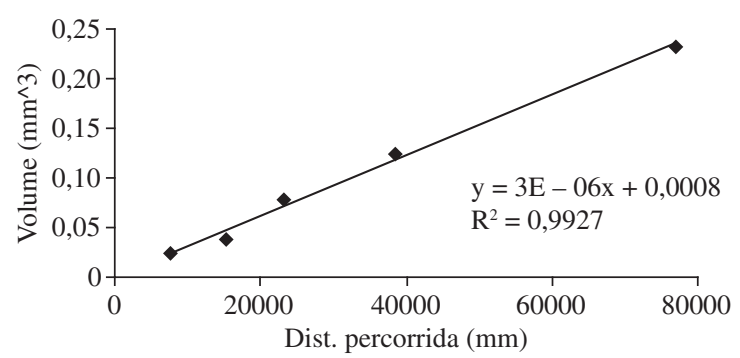

Figura 3. Taxa de desgaste da amostra 1.

Tabela 3. Valores dos coeficientes de atrito e dos coeficientes de desgaste microabrasivo das amostras recobertas.

\begin{tabular}{ccc}
\hline Amostra & Coef. atrito $(\mu)$ & Coef. desgaste $(\mathbf{K})$ \\
\hline 1 & 0,040 & $2,95 \times 10^{-5}$ \\
2 & 0,050 & $2,90 \times 10^{-5}$ \\
3 & 0,040 & $2,02 \times 10^{-5}$ \\
4 & 0,040 & $3,85 \times 10^{-5}$ \\
5 & 0,035 & $1,28 \times 10^{-5}$ \\
6 & 0,035 & $2,32 \times 10^{-5}$ \\
7 & 0,050 & $1,60 \times 10^{-5}$ \\
8 & 0,035 & $3,23 \times 10^{-5}$ \\
Lâmina de PET & 0,1 & $3,0 \times 10^{-5}$ \\
\hline
\end{tabular}


(PTFE), polipropileno (PP) e PEEK na ordem de $10^{-5} \mathrm{~mm}^{3} / \mathrm{m}$. Palabiyik e Bahadur ${ }^{[14]}$ citam valores de $4,33 \times 10^{-5} \mathrm{~mm}^{3} / \mathrm{N} . \mathrm{mm}$ para o coeficiente de desgaste de polietileno de alta densidade (HDPE).

A Figura 4 representa a sequiência das crateras formadas durante o ensaio de caloteste da amostra 1 indicando a distância percorrida com 1000, 500 e 300 voltas, respectivamente.

A Figura 5 ilustra a perfilometria 3D de uma cratera de 500 voltas com um diâmetro de $1,86 \mathrm{~mm}$, no recobrimento de PET.

A análise dos resultados dos experimentos é apresentada com base no efeito dos fatores e suas interações. As interações PG (pressão X gás), PA (pressão X taxa de alimentação) e GA (gás $x$ taxa de alimentação) são apresentadas em gráficos

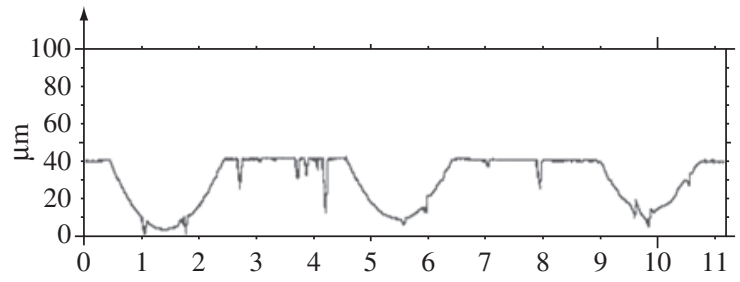

Figura 4. Crateras formadas na amostra 1, na seqüência de 1000,500 e 200 voltas.

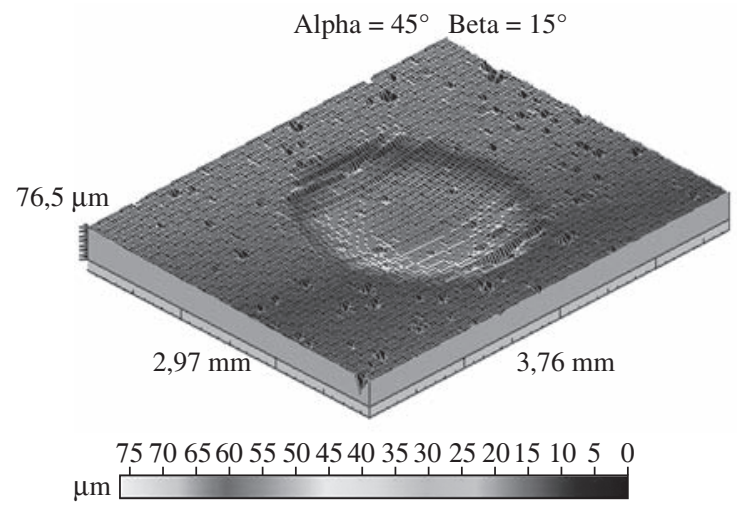

Figura 5. Perfilometria de uma cratera.

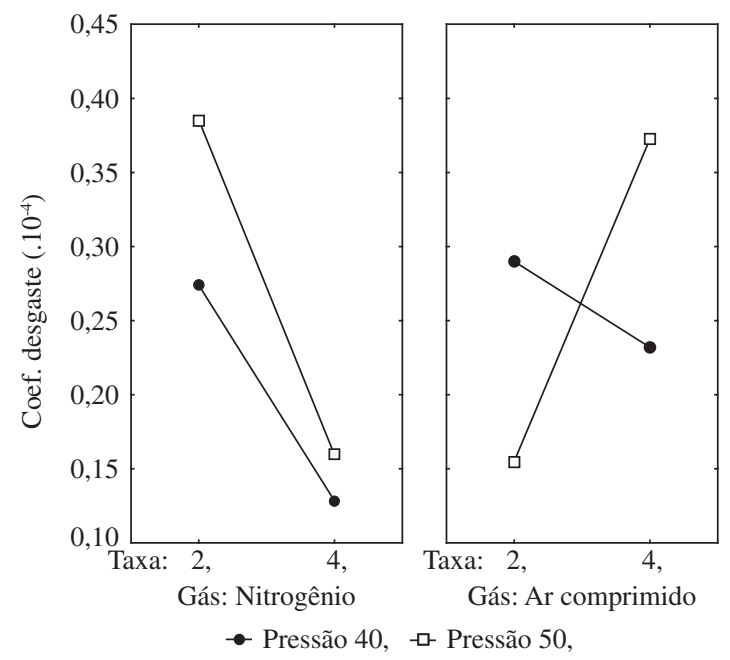

Figura 6. Interação dos fatores no coeficiente de desgaste. de interações, Figura 6, sugerindo serem relevantes. O desvio padrão dos resultados de desgaste foi de $9,68 \times 10^{-6}$.

Para a interação GA, menores valores do coeficiente de desgaste são alcançados quando se usa nitrogênio como gás de arraste e uma maior taxa de alimentação. Utilizando-se taxas de alimentação menores, o efeito do ar comprimido como gás de arraste proporcionou menores coeficientes de desgaste. Para as interações PA e PG, resultados significativos são destacados no nível baixo de pressão.

Com um Gráfico de Pareto (Figura 7) a estimativa para cada efeito é apresentada em relação ao coeficiente de desgaste. As interações de $2^{\mathrm{a}}$ e $3^{\mathrm{a}}$ ordens são todas significativas. Dos efeitos principais a variável tipo de gás de arraste $(G)$ não foi significante, já que se encontra a esquerda da linha do valor da probabilidade de significância $(\mathrm{p}=0,05)$.

Os parâmetros de processo da aspersão têm efeito significativo sobre as partículas aspergidas na chama exercendo influência sobre a resistência ao desgaste no recobrimento formado. De acordo com cálculos estequiométricos da chama, o efeito destes fatores pode ser explicado, na quantidade de ar comprimido ou nitrogênio que participam da queima afetando a temperatura da chama, consequentemente a fusão das partículas no vôo durante o recobrimento. Um aumento da pressão dos gases implica também em um aumento da faixa de inflamabilidade do combustível gasoso, resultando em um aumento da concentração dos reagentes. Esta variação na dinâmica dos gases pode ocasionar mudanças na microestrutura dos recobrimentos.

$\mathrm{O}$ efeito da rugosidade dos filmes também foi observado nos resultados de desgaste e atrito. Kato ${ }^{[15]}$ mostra que menores coeficientes de atrito são obtidos em menores rugosidades.

Nos ensaios de PSD, notou-se que uma rugosidade de 3,0 $\mu \mathrm{m}$, promove uma variação no coeficiente de atrito de 0,03 a 0,07 (Figura 8). Zhang ${ }^{[16]}$ relata que o efeito da rugosidade não influencia a média do coeficiente de atrito nos polímeros.

Optou-se por utilizar-se amostras polidas, com baixa rugosidade superficial, $(0,5 \pm 0,02 \mu \mathrm{m})$, a fim de se diminuir a variação do coeficiente de atrito, 0,03 e 0,05 (Figura 9). Unal et $\mathrm{al}^{[17]}$ apresentam valores de coeficientes de atrito, para teflon (PTFE), de 0,06.

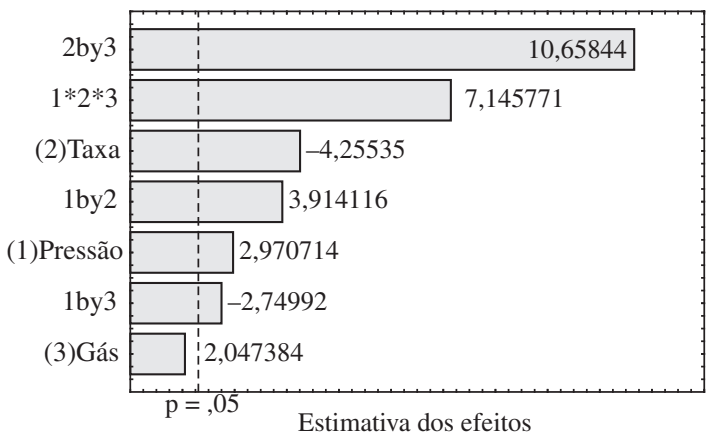

Figura 7. Gráfico de Pareto para estimativa dos efeitos das variáveis no coeficiente de desgaste. 


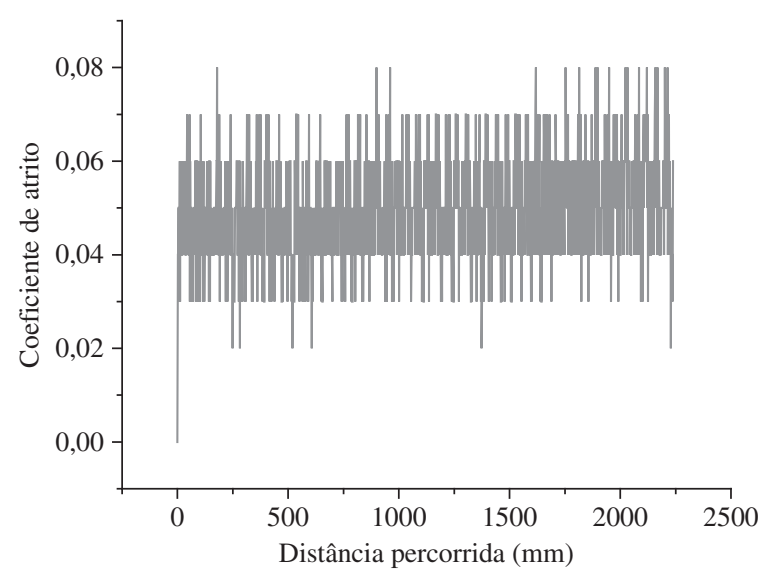

Figura 8. Amostra com rugosidade superficial de $3,0 \mu \mathrm{m}$.

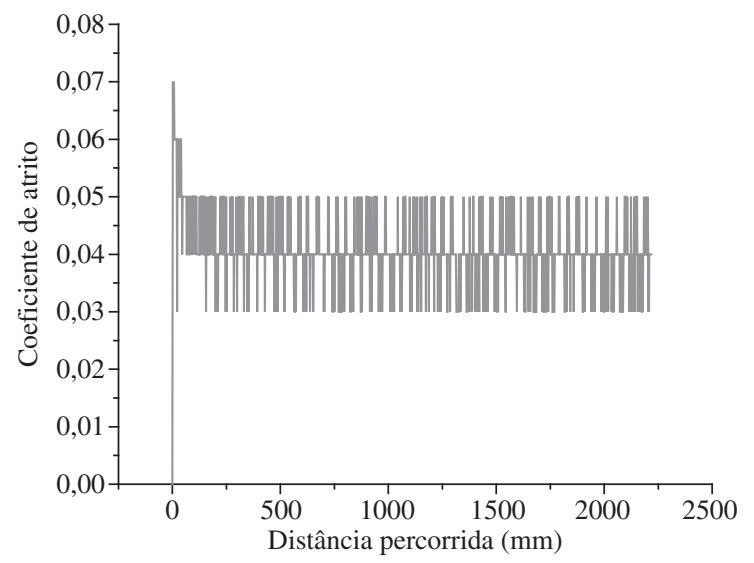

Figura 9. Amostra polida, rugosidade de $0,5 \mu \mathrm{m}$.

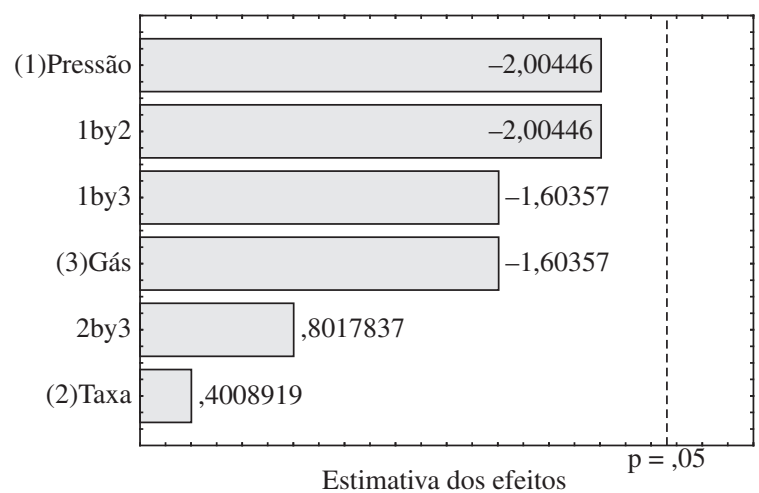

Figura 10. Gráfico de Pareto para estimativa dos efeitos dos fatores no coeficiente de atrito.

A Figura 10 representa o Gráfico de Pareto da estimativa dos efeitos dos parâmetros do processo nos valores do coeficiente de atrito dos recobrimentos obtidos no ensaio de PSD. O efeito principal dos fatores pressão, taxa e gás, e suas interações, não mostraram ser significativos, com valores de probabilidade de significância maiores que 0,05 . Estes resultados podem estar relacionados ao efeito viscoelástico.

Zhang ${ }^{[16]}$ relata a dureza como importante papel no desempenho do atrito. Atrito em PTFE no estágio inicial do deslizamento tem apresentado uma enorme dependência da

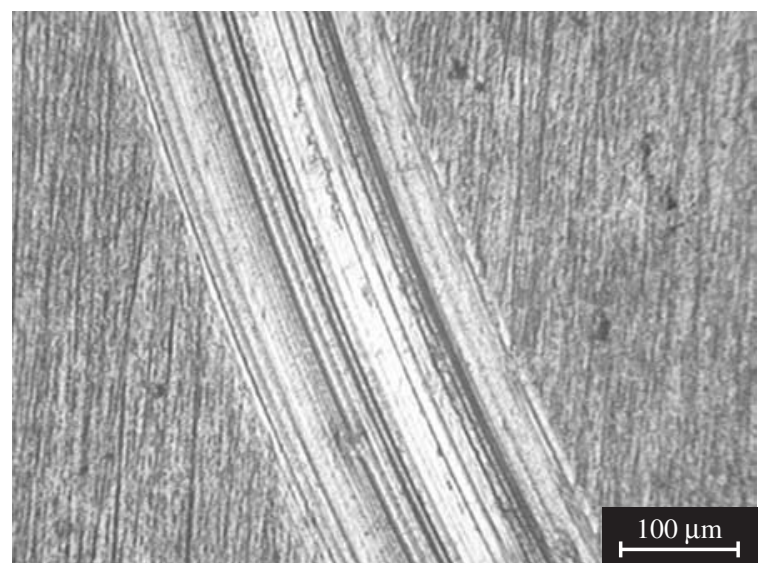

Figura 11. Trilha de um ensaio de PSD

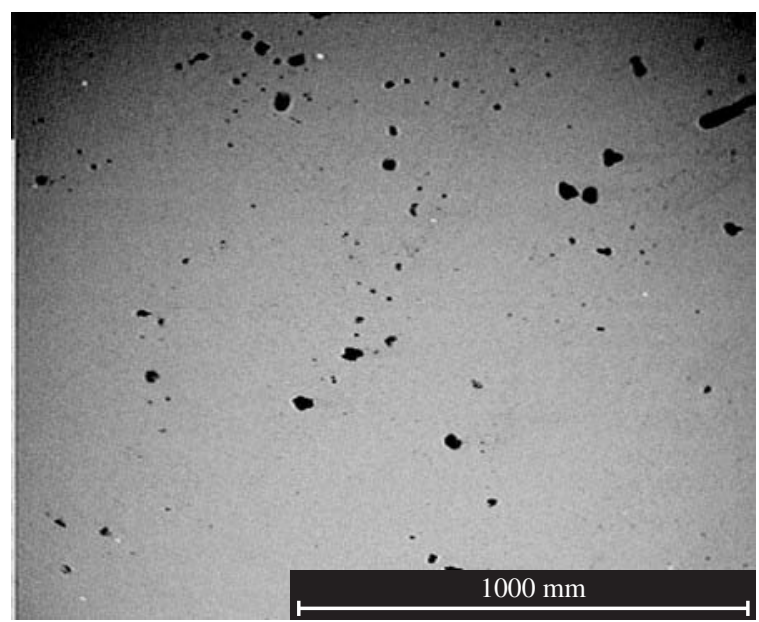

Figura 12. Partículas de PET na superfície da esfera.

estrutura cinético-molecular na zona de contato. $\mathrm{O}$ atrito pode ter origem viscoelástica sob certas condições de testes.

Nota-se este efeito nos ensaios realizados, prevalecendo um amassamento do filme na superfície de contato, com visualização da trilha percorrida (Figura 11), mas com valores de desgaste insignificantes.

Com o objetivo de identificar uma transferência de massa para a esfera de aço-cromo, usada no ensaio de pino sobre disco, as amostras foram examinadas ao microscópio eletrônico de varredura (MEV). Para cada ensaio utilizou-se uma única esfera de aço cromo.

A Figura 12 mostra a presença de partículas de PET na superfície da esfera representada pelos pontos escuros.

\section{Conclusão}

Os coeficientes de desgaste encontrados são relativamente baixos, da ordem de $10^{-5}$, próximos aos de lâminas de garrafas PET $\left(3,0 \times 10^{-5}\right)$.

De acordo com as análises do planejamento de experimentos, para a resposta coeficiente de desgaste os efeitos das interações de $2^{\mathrm{a}}$ e $3^{\mathrm{a}}$ ordens foram significativos, indicando que as três variáveis analisadas, pressão dos gases de combustão, taxa de alimentação e tipo de gás de arraste do ali- 
mentador afetam a resistência ao desgaste do filme. Menores valores do coeficiente de desgaste foram encontrados utilizando-se pressão dos gases de 50 psi, taxa de alimentação de $2 \mathrm{~g} / \mathrm{min}$ e ar comprimido como gás de arraste.

Durante os ensaios de PSD, as amostras previamente polidas apresentaram menores resultados de coeficientes de atrito, aproximadamente 0,04 , obtendo-se menores coeficientes de atrito para menores rugosidades. Constatou-se uma transferência de massa do filme de PET para a superfície da esfera de aço-cromo na forma de pequenas partículas irregulares.

Verificou-se que os efeitos principais da pressão dos gases, taxa de alimentação e tipo de gás de arraste, e suas interações nos coeficientes de atrito não são significativos.

\section{Agradecimentos}

CAPES pelo auxílio à bolsa, aos Laboratórios de Metalografia e Tribologia do CETEC-MG.

\section{Referências Bibliográficas}

1. Herman, H. \& Sampath, S. - Thermal Spray Coatings (2005).

2. Brogan, J. A. - Processing and Property Relationships of Thermally Sprayed Polymer Systems, New York: State University of New York at Stony Brook. (PhD Thesis) (1996).

3. Brogan, J. A. - MRS Bulletin (2000).

4. Bao, Y. \& Gawne, D. T. - Effect of Processing on the Wear Resistance of Thermally Sprayed Epoxy Coatings, ASM International, Materials Park, Ohio, USA (1996).

5. Petrovicova, E. \& Schadler, L. S. - International Materials Reviews, 47, 4 (2002).

6. Zhang, T.; Gawne, D. T. \& Bao, Y. - The Effect of Deposition Parameters on The Degradation of Plasma
Sprayed Polymer Coatings, Thermal Spray: Pratical Solutions for Engineering Problems, Edited by C. C. Berndt, ASM International, Ohio, p.231-237 (1996).

7. Briscoe, B. J. - Materials aspects of polymer wear, Scripta Metalurgica et Material, 24, p.839-844, (1990).

8. Lancaster, J. K. - Wear, 141, p.159-183, (1990).

9. Hutchings, I. M. - Tribology: Friction and Wear of Engineering Materials, Edward Arnold, London, p.51, (1992).

10. Briscoe B. J. \& Sinha, S. K. - Wear of Polymers, Proceedings of the Institution of Mechanical Engineers, 216 (J6), p.401-413, (2002).

11. Branco, J. R. T.; Campos, S. R. V.; Duarte, L. T. \& Lins, V. F. C. - Journal of Applied polymer Science, (2004).

12. Shipway, P.H.; Ngao, N.K. - Wear, 255, p.742-750 (2003).

13. Friedrich, K.; Reinicke, R. \& Zhang, Z. - Wear of polymer composites, Proc Instn Engrs, 216 Part J: Engineering Tribology (2002).

14. Palabiyik, M. \& Bahadur, S. - Tribological studies of polyamide 6 and high-density polyethylene blends filled with PTFE and cooper oxide and reinforced with short glass fibers, Wear, 253, p.369-376 (2002).

15. Kato, K. - Wear in relation to friction - a review, Wear, 241, p.151-157, (2000).

16. Zhang, S. W. - State-of-art of polymer tribology, Tribology International, 31, 1-3, p.49-60 (1998).

17. Unal, U. \& Mimaroglu, A. - Influence of test conditions on the properties of polymers, Industrial Lubrification and Tribology (2003).

Enviado: 12/10/06

Reenviado:26/03/07

Aceito: 04/04/07 